\title{
The M 16 molecular complex under the influence of NGC 6611
}

\section{Herschel's perspective of the heating effect on the Eagle Nebula ${ }^{\star} \star \star$}

T. Hill ${ }^{1}$, F. Motte ${ }^{1}$, P. Didelon ${ }^{1}$, G. J. White ${ }^{2,3}$, A. P. Marston ${ }^{4}$, Q. Nguyễn Lương ${ }^{1}$, S. Bontemps ${ }^{5}$, Ph. André ${ }^{1}$, N. Schneider ${ }^{1}$, M. Hennemann ${ }^{1}$, M. Sauvage ${ }^{1}$, J. Di Francesco ${ }^{6}$, V. Minier ${ }^{1}$, L. D. Anderson ${ }^{7}$, J. P. Bernard ${ }^{8}$, D. Elia ${ }^{9}$, M. J. Griffin ${ }^{10}$, J. Z. Li ${ }^{11}$, N. Peretto ${ }^{1}$, S. Pezzuto ${ }^{9}$, D. Polychroni ${ }^{9}$, H. Roussel ${ }^{12}$, K. L. J. Rygl ${ }^{9}$, E. Schisano ${ }^{9}$, T. Sousbie ${ }^{12}$, L. Testi ${ }^{13}$, D. Ward Thompson ${ }^{10,14}$, and A. Zavagno ${ }^{15}$

${ }^{1}$ Laboratoire AIM, CEA/IRFU CNRS/INSU Université Paris Diderot, CEA-Saclay, 91191 Gif-sur-Yvette Cedex, France e-mail: tracey.hill@cea.fr

2 The Rutherford Appleton Laboratory, Chilton, Didcot, OX11 0NL, UK

3 Department of Physics and Astronomy, The Open University, Milton Keynes, UK

${ }^{4}$ Herschel Science Centre, ESAC, Spain

5 Université de Bordeaux, OASU, Bordeaux, France

${ }^{6}$ National Research Council of Canada, 5071 West Saanich Road, Victoria, BC, V9E 2E7, Canada

7 Department of Physics, West Virginia University, Morgantown, WV 26506, USA

8 Université de Toulouse, UPS, CESR, 9 avenue du Colonel Roche, CNRS, UMR 5187, 31028 Toulouse Cedex 4, France

9 IAPS - Instituto di Astrofisica e Planetologia Spaziali, via Fosso del Cavaliere 100, 00133 Roma, Italy

10 School of Physics and Astronomy, Cardiff University, Queens Buildings, The Parade, Cardiff, CF243AA, UK

11 National Astronomical Observatories, Chinese Academy of Sciences, A20 Datun Road, Chaoyang District, Beijing 100012, PR China

12 Institut d'Astrophysique de Paris, Université Pierre et Marie Curie (UPMC), CNRS (UMR 7095), 75014 Paris, France

13 ESO, Karl Schwarzschild str. 2, 85748 Garching bei Munchen, Germany

14 Jeremiah Horrocks Institute, University of Central Lancashire, PR1 2HE, UK

15 Laboratoire d'Astrophysique de Marseille UMR 6110, CNRS, Université de Provence, 38 rue F. Joliot-Curie, 13388 Marseille, France

Received 10 February 2012 / Accepted 26 April 2012

\section{ABSTRACT}

\begin{abstract}
We present Herschel images from the HOBYS key program of the Eagle Nebula (M 16) in the far-infrared and sub-millimetre, using the PACS and SPIRE cameras at $70 \mu \mathrm{m}, 160 \mu \mathrm{m}, 250 \mu \mathrm{m}, 350 \mu \mathrm{m}, 500 \mu \mathrm{m}$. M 16, home to the Pillars of Creation, is largely under the influence of the nearby NGC 6611 high-mass star cluster. The Herschel images reveal a clear dust temperature gradient running away from the centre of the cavity carved by the OB cluster. We investigate the heating effect of NGC 6611 on the entire M 16 star-forming complex seen by Herschel including the diffuse cloud environment and the dense filamentary structures identified in this region. In addition, we interpret the three-dimensional geometry of M 16 with respect to the nebula, its surrounding environment, and the NGC 6611 cavity. The dust temperature and column density maps reveal a prominent eastern filament running north-south and away from the high-mass star-forming central region and the NGC 6611 cluster, as well as a northern filament which extends around and away from the cluster. The dust temperature in each of these filaments decreases with increasing distance from the NGC 6611 cluster, indicating a heating penetration depth of $\sim 10 \mathrm{pc}$ in each direction in $3-6 \times 10^{22} \mathrm{~cm}^{-2}$ column density filaments. We show that in high-mass star-forming regions OB clusters impact the temperature of future star-forming sites, modifying the initial conditions for collapse and effecting the evolutionary criteria of protostars developed from spectral energy distributions. Possible scenarios for the origin of the morphology seen in this region are discussed, including a western equivalent to the eastern filament, which was destroyed by the creation of the OB cluster and its subsequent winds and radiation.
\end{abstract}

Key words. ISM: clouds - ISM: structure - dust, extinction - stars: early-type - stars: protostars

\section{Introduction}

Forming in dynamic and ever-changing environments, highmass stars are complex entities which, through radiation and stellar winds, heavily impact their natal environments, possibly even triggering the formation of new stars and planetary systems. A general rarity of candidates and their rapid evolutions mean

\footnotetext{
* Herschel is a ESA space observatory with science instruments provided by European-led Principal Investigator consortia and with important participation from NASA.

$\star \star$ Appendices are available in electronic form at

http://www . aanda.org
}

that it is difficult to characterise the early evolutionary stages of high-mass stars (cf. Garay \& Lizano 1999).

The Herschel Space Observatory (launched 2009; Pilbratt et al. 2010) has already had a significant impact on star formation studies, owing primarily to the large key programs devoted to this field, specifically the Herschel imaging study of OB Young Stellar objects (HOBYS; Motte et al. 2010) and Gould Belt Survey (André et al. 2010), as well as the Hi-Gal survey (Molinari et al. 2010). These programs target the natal environments of burgeoning young stars, such as interstellar filaments and ridges (Arzoumanian et al. 2011; Hill et al. 2011), as well as sources embedded within them, e.g., prestellar cores 
and protostars (Bontemps et al. 2010; Hennemann et al. 2010; Giannini et al. 2012), and investigate the impact of OB stars on cloud formation (Zavagno et al. 2010; Schneider et al. 2010). The primary HOBYS ${ }^{1}$ driver is to compile an unbiased census of high-mass star progenitors, and their natal ridges, in the nearest ( $<3 \mathrm{kpc}$ ) star-forming complexes (Motte et al. 2010), one of which is M 16.

The Eagle Nebula (M 16) is a young $\left(1-3 \times 10^{6} \mathrm{yr}\right.$; Hillenbrand et al. 1993), active high-mass star-forming region in the constellation of Serpens $\sim 2 \mathrm{kpc}$ from the Sun. M 16 was first observed by de Cheseaux in 1745 and then rediscovered by Messier in 1764 who was the first to comment on its nebulosity. The European Space Agency's (ESA's) Infrared Space Observatory (ISO; Cesarsky et al. 1996) revealed the presence of unusually hot dust in the core of the nebula (Pilbratt et al. $1998, T_{\text {colour }} \sim 200 \mathrm{~K}$ ). But it was the Hubble Space Telescope images of its ionised gas (Hester et al. 1996), better known as the "Pillars of Creation", that has made M 16 iconic and one of the most observed objects in the sky.

M 16, and especially these pillars, has since been observed at many wavelengths spanning the electromagnetic spectrum, as well as in many dedicated spectral line studies (e.g., Pound 1998; White et al. 1999; Allen et al. 1999; Urquhart et al. 2003; Linsky et al. 2007, to name a few). More recently, M 16 was observed in the mid-infrared with IRAC $(3.6-8.0 \mu \mathrm{m})$ as part of the Spitzer GLIMPSE legacy program (Indebetouw et al. 2007), and in the far-infrared with MIPS $(24 \mu \mathrm{m}$ and $70 \mu \mathrm{m})$ as part of the Spitzer MIPSGAL program (Flagey et al. 2011). The former survey targeted the warm dust towards this region and revealed its (large) population of young stellar objects (YSOs) that these authors deemed protostellar candidates. The latter paper examined the dust emission and composition, such as the grain type and size, in this region, and concluded that a supernova remnant may be responsible for heating the inner portion of M 16.

Adjacent to the Eagle Nebula, and responsible for heating and ionising the Nebula, is the young open star cluster NGC 6611. At a distance of $\sim 1.75-2 \mathrm{kpc}$ (Gvaramadze \& Bomans 2008), NGC 6611 contains four early-type O stars ( 2-3 Myr; Dufton et al. 2006), and is also associated with the cluster of a B2.5I star ( $\sim 6 \mathrm{Myr}$; Hillenbrand et al. 1993). The total stellar mass of the NGC 6611 cluster is $\sim 2 \times 10^{4} M_{\odot}$ (Wolff et al. 2007). See Gvaramadze \& Bomans (2008) and Belikov et al. (2000) for more information on the NGC 6611 cluster.

We present here unprecedented high-resolution highsensitivity Herschel observations, spanning the far-infrared and submillimetre regime, of the Eagle Nebula. These Herschel data provide unique access to this far-infrared regime at much greater spatial resolution, and additionally cover a greater area around the central NGC 6611 cluster and the Pillars of Creation, than previous (space) observations and studies. Herschel allows us to probe the cold component of the dust, complementary to Spitzer, and to deepen the study of warm dust (through the $70 \mu \mathrm{m}$ band) in this region. Here, we present a structural analysis of the entire M 16 molecular cloud complex, with respect to the well-known central OB cluster. A companion paper by White et al. (in prep.) examines the protostellar content of the M 16 region as detected by Herschel.

\section{Observations and data reduction}

M 16 was observed on 2010 March 24 and 2010 September 1112, as part of the HOBYS key program (Motte et al. 2010).

\footnotetext{
1 http://www.herschel. fr/cea/hobys/en/
}

In March, the SPIRE $(250 \mu \mathrm{m}, 350 \mu \mathrm{m}, 500 \mu \mathrm{m}$; Griffin et al. 2010) camera was inadvertently turned off and thus only the shorter PACS (70, $160 \mu \mathrm{m}$; Poglitsch et al. 2010) wavelengths were mapped during this period. In September, the parallel-scan mode of Herschel was used to map simultaneously with both instruments at the aforementioned five bands, using the slow scan speed $\left(20^{\prime \prime} / \mathrm{s}\right)$. For these September observations, the SPIRE camera was aligned with the PACS data taken from March, allowing 100\% common area coverage (normally, the positional offset between the PACS and SPIRE instruments results in a small $\left(20^{\prime}\right)$ spatial offset between the PACS and SPIRE maps), as well as an extended PACS coverage to the east and south (see Fig. A.1, top). A total area of $\sim 1.5 \mathrm{deg}$ by $1.5 \mathrm{deg}$ was mapped using two orthogonal scan directions.

Each of the March and September data were reduced with version 5.0.1975 of the Herschel Interactive Processing Environment (HIPE ${ }^{2}$; Ott 2010) adopting standard steps of the default pipeline to Level 1 products including calibration and deglitching. To improve the baseline subtraction, data taken during the turn-around of the telescope were used. Calibration of the PACS data has been found to be within 5\%, whilst SPIRE calibration is within $10 \%$ for all bands (see the respective observers' manuals).

Maps were produced using the HIPE Level 1 data and v7 of the Scanamorphos software package ${ }^{3}$ which performs baseline and drift removal before regriding (Roussel 2012). The two sets of PACS data, from March and September, were combined within Scanamorphos using the "/galactic" option, to produce a single map of the entire PACS coverage. For simplicity, we hereafter use PACS data to refer to the combination of the two individual PACS observations, rather than referring to one particular data set. The individual Herschel images at each wavelength can be found in Fig. A.1, including the highest resolution $70 \mu \mathrm{m}$ image which traces the hot dust and protostars in this region. The three colour image of the the entire M 16 complex, as well as a zoom to the central region around the OB cluster is given in Fig. 1.

\section{Structural analysis}

The Herschel maps of the Eagle Nebula show bright emission throughout, particularly around the NGC 6611 cluster (see Figs. 1 and A.1). This bright emission covers the Pillars of Creation and the Spire ${ }^{4}$ east of the cluster and a possible ridge (see Sect. 3.3) of material to the north-west.

Immediately to the north of this ridge is a prominent archlike structure (see Fig. 1, left). While weak diffuse emission can be seen towards this arch-like structure in the infrared $(8 \mu \mathrm{m}$; see Fig. 5 of Indebetouw et al. 2007), it is much more visually striking and associated with a clear density enhancement in our Herschel images e.g., Fig. 1, right (see also Figs. 2 and 3 below). To the south-west of the ridge is the massive YSO (MYSO) identified by Indebetouw et al. (2007, also known as IRAS 18152-1346) as the most luminous YSO $\left(1 \times 10^{3} L_{\odot}\right)$ in the region. The Herschel images of M 16 indicate many other sites of star formation, such as small clusters of sources in the south-east of the map, which are more easily identified in the higher resolution PACS data (see Fig. A.1, top).

\footnotetext{
2 http://herschel.esac.esa.int/HIPE_download.shtml

http://www2 . iap . fr/users/roussel/herschel/index .html

4 The Spire is a well known region in the Eagle Nebula. See, http://en.wikipedia.org/wiki/Messier_16
} 

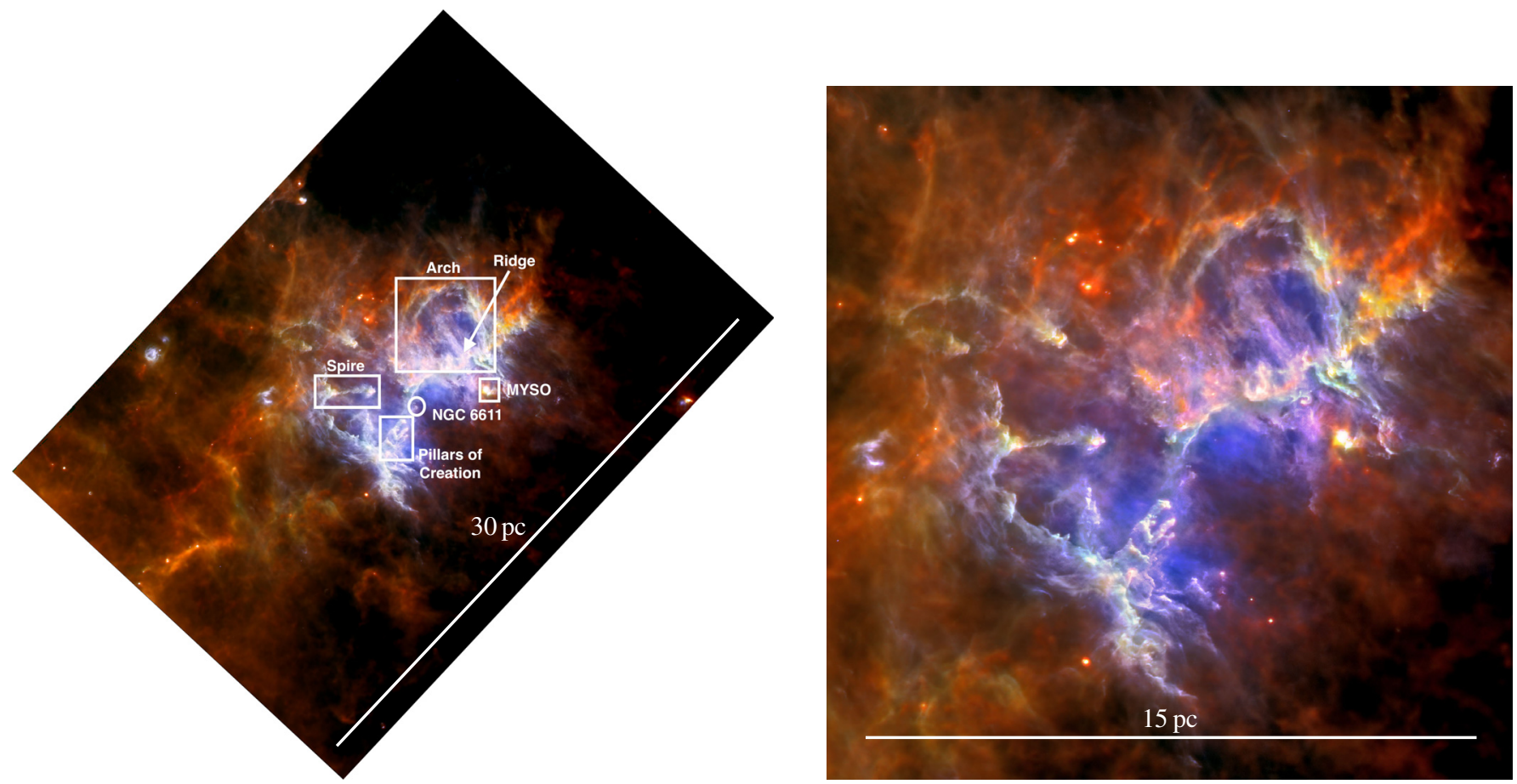

Fig. 1. Three-colour image of M 16 using $70 \mu \mathrm{m}$ (blue), $160 \mu \mathrm{m}$ (green), $250 \mu \mathrm{m}$ (red). Left: image of the entire M 16 complex with well-known regions from the literature, as well as regions identified (the arch and ridge features) and discussed in this paper, indicated on the map. Right: zoom to the central region of M 16 (see left image).
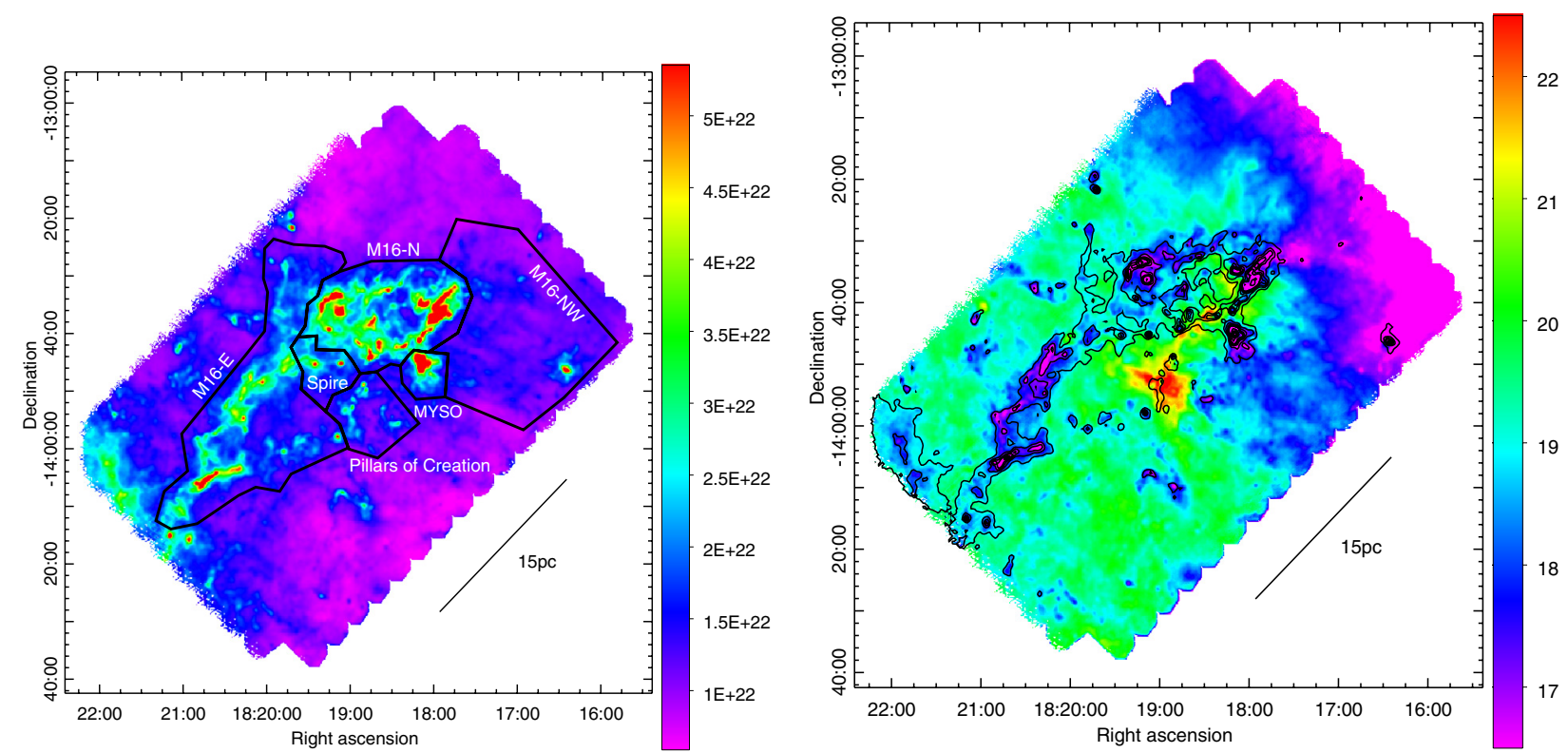

Fig. 2. Left: column density map of M 16 (37" resolution). Right: dust temperature map, with the column density contours (left) overlaid (37" resolution). The contours are $2.0,3.1,4.3,5.4,6.5 \times 10^{22}, 1.1,1.6,2.0,2.5 \times 10^{23} \mathrm{~cm}^{-2}$.

The three-colour composite image of this region (Fig. 1) shows a very distinct temperature gradient away from the NGC 6611 cluster. This temperature gradient is reminiscent of that seen in other HOBYS regions such as RCW 120 (Zavagno et al. 2010) and the bubble HiI region study of Anderson et al. (2012).

\subsection{Column density and dust temperature maps}

The dust temperature and column density $\left(N_{\mathrm{H}_{2}}\right)$ maps of M 16 were drawn by fitting, pixel-by-pixel, spectral energy distributions (SEDs) using a modified blackbody model (Hill et al. 2009, 2010), following the procedure outlined by Hill et al. (2011). These data were convolved to the resolution of the $500 \mu \mathrm{m}$ band (37"), and the zero offsets, which were determined from comparison with Planck and IRAS following the procedure detailed by Bernard et al. (2010), were applied to the individual maps prior to fitting. Only the four longest Herschel wavebands, which trace cold dense material, were used to make the column density and dust temperature maps (Fig. 2), a method consistent within the HOBYS programme (Hill et al. 2011; Nguyen Luong et al. 2011). The $70 \mu \mathrm{m}$ emission is excluded from the 


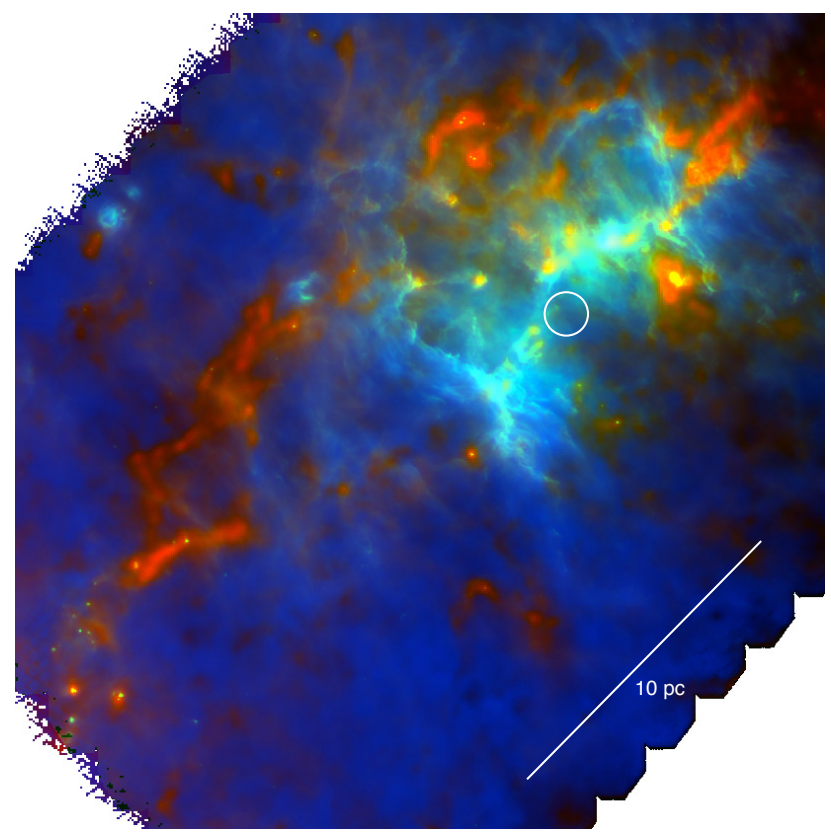

Fig. 3. Three colour composite of the column density (red), dust temperature (blue) and $70 \mu \mathrm{m}$ (green) images. The white circle indicates the location of the NGC 6611 cluster. The extent of the influence of the photodissociation region, created by the presence of the OB cluster, is clearly highlighted in green at the centre of the map. Limb-brightening effects occur here around column density peaks and on many low column density filaments around the OB cluster.

fits as it is not tracing the cold dust that we are most interested in, and is likely tracing small grains in hot PDRs instead (cf. Fig. 3). Furthermore, excluding the $70 \mu \mathrm{m}$ emission ensures that the SED fit is more robust, since the $70 \mu \mathrm{m}$ flux does not completely fit a single temperature greybody model dominated by longer wavelength fluxes. We used a dust opacity law in which the dust opacity per unit mass column density $\left(\kappa_{v}\right)$, is given by $\kappa_{v}=0.1(v / 1000 \mathrm{GHz})^{\beta} \mathrm{cm}^{2} / \mathrm{g}$ (e.g. Motte et al. 2010), and a dust spectral index of $\beta=2$. The latter was fixed in line with the literature (Goldsmith et al. 1997, and references therein) and because we do not have longer wavelength (sub)millimetre data to constrain $\beta$ accurately. The quality of a SED fit was assessed using $\chi^{2}$ minimisation. Most of the individual single temperature SED fits comprising the dust temperature and column density maps peaked around, or longward of, the $160 \mu \mathrm{m}$ flux the shortest wavelength data used in the fit. The observed rms of cirrus noise in the derived column density map is $\sim 2 \times 10^{21} \mathrm{~cm}^{-2}$ $\left(A_{\mathrm{V}} \sim 2 \mathrm{mag}\right)$. Note however, that the column density and dust temperature are line of sight measures.

The median temperature throughout M 16 is $\sim 18 \mathrm{~K}$, with slightly cooler temperatures in the northern part of the Herschel map, which is furthest from the Galactic plane. The eastern portion of M 16 is dominated by a dense filament $\left(3-7 \times 10^{22} \mathrm{~cm}^{-2}\right)$ situated directly east of, and running parallel to the Pillars of Creation. This "Eastern Filament" appears as a cool temperature depression ( $\sim 16 \mathrm{~K}$; Fig. 2, right, see Sect. 4.1) with respect to the warmer surrounding material.

Figure 3, a three colour composite of the column density, dust temperature and $70 \mu \mathrm{m}$ image, highlights the region affected by strong PDR emission, i.e., the interface between the cloud and the cavity swept out by the NGC 6611 cluster. The inclusion of the $70 \mu \mathrm{m}$ emission here highlights clear examples of limb brightening, where the filamentary structures around

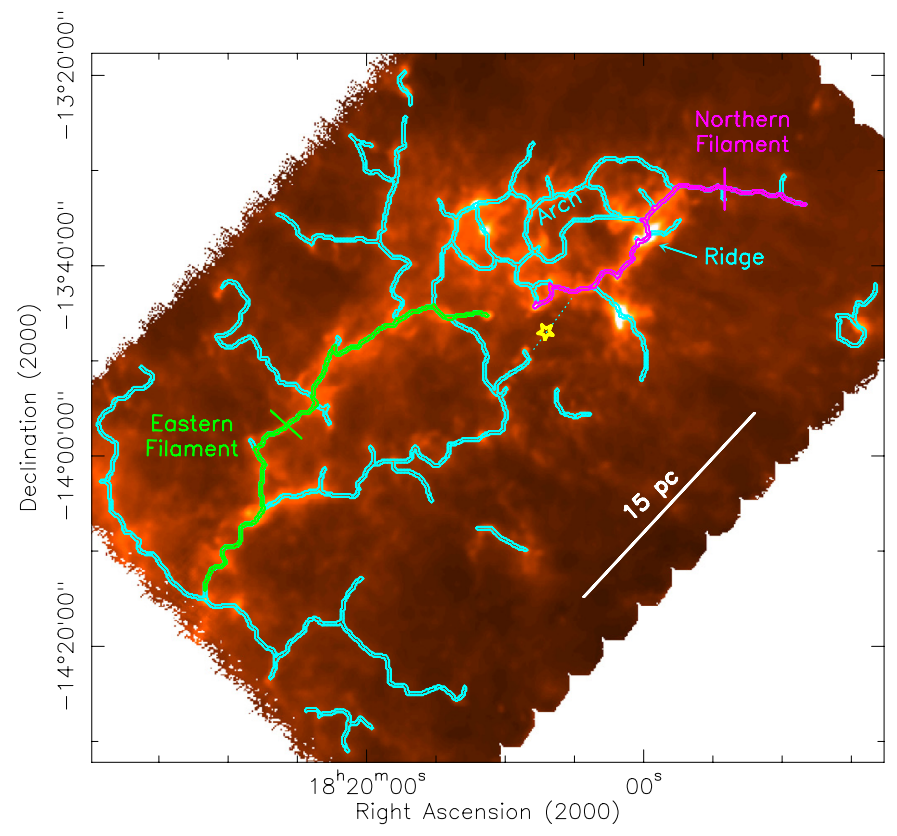

Fig. 4. Filaments (cyan) in M 16 detected by DisPerSE overlaid on the column density map. The Eastern Filament is shown here in green, whilst the Northern Filament is in magenta. The position of the NGC 6611 cluster is depicted by the yellow star. The temperature minimum of the Eastern Filament and the end of the temperature gradient for the Northern Filament, seen in Fig. 5, are indicated by lines perpendicular to the respective filaments. The possible Western Filament (see Sect. 5.1) runs parallel to the Eastern Filament, and the possible continuation to the Northern Filament is indicated by a dashed line.

the cavity have heated edges at the ionisation front created by the NGC 6611 cluster. Furthermore, this Figure demonstrates the strong correspondence between high column density and low temperature, especially with respect to the Eastern Filament (clearly visible here in red).

\subsection{Filamentary structure}

Recent Herschel observations have revealed the ubiquity of interstellar filaments, especially in star-forming regions. André et al. $(2010,2011)$ proposed that in low-mass star-forming regions only those filaments above an $A_{\mathrm{V}}$ of $\sim 7 \mathrm{mag}$ are gravitationally unstable (supercritical) and thus capable of forming prestellar cores in their interiors. In fact, a large portion of the molecular complexes observed as part of HOBYS are above this star-forming threshold (e.g., Hill et al. 2011; Nguyen Luong et al. 2011; Schneider et al. 2012).

To take a census of the filaments in the M 16 star-forming complex, the DisPerSE algorithm (Sousbie 2011; Sousbie et al. 2011) was applied to the column density map. DisPerSE works with Morse theory and the concept of persistence to identify topological structures (see Sousbie 2011, for a detailed explanation of these concepts) such as filaments and ridges, and connect their saddle-points with maxima by integral lines. This algorithm has already been successfully applied by our group to trace filamentary structures in star-forming regions (e.g., see Hill et al. 2011; Peretto et al. 2012). The crest points of the filaments, as identified by DisPerSE are presented in Fig. 4.

The southern half of M 16 is dominated by two main filaments which run roughly parallel to each other near the centre of the Herschel map, before converging to a solitary filament 

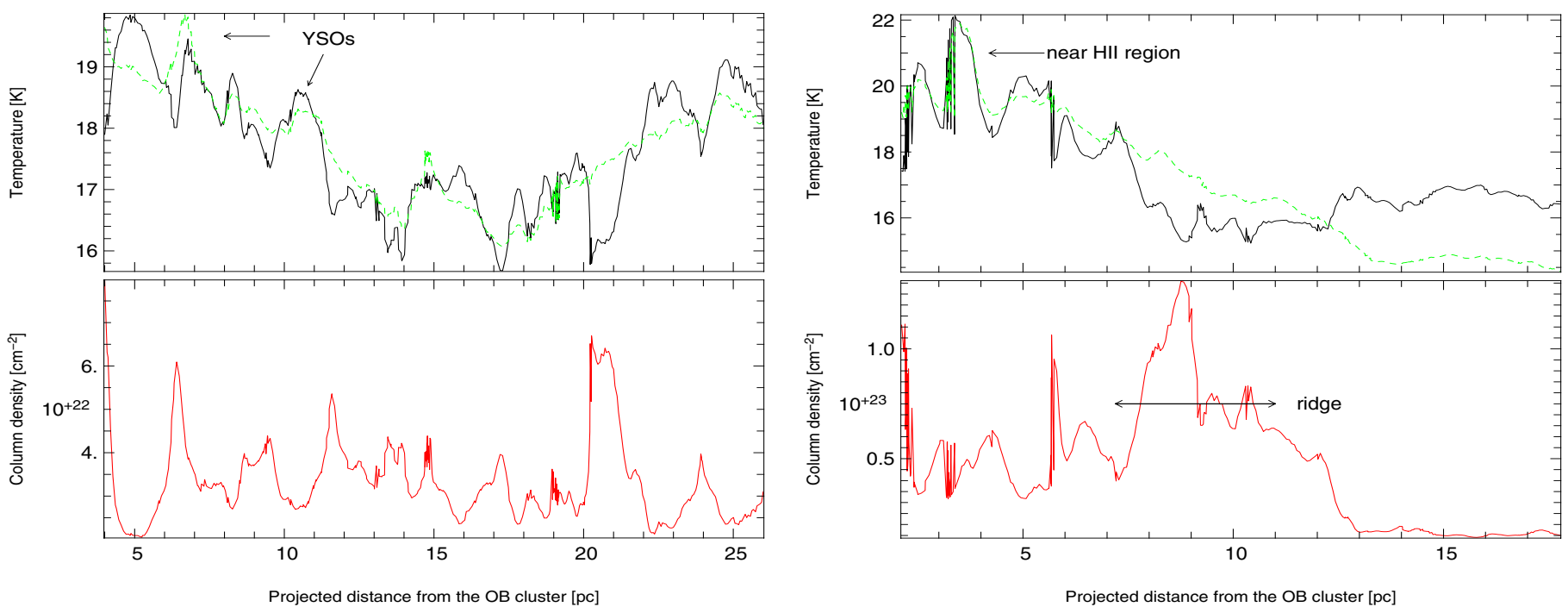

Fig. 5. The dust temperature (top) and column density (bottom) profiles along the Eastern (left) and Northern (right) Filaments. The positions along the filaments are plotted as a function of their projected distance from the cluster. The NGC 6611 cluster is located at " 0 " on the $x$-axis, centred at $1818.8-13$ 49. The green dashed line on the dust temperature (top) is the dust temperature after removing the effects of cold sources within the filaments, following the relation of Juvela \& Ysard (2011; Fig. 1, see footnote 6 and also Didelon et al., in prep., who examine this relation for Herschel filaments). The thick vertical lines seen on the profile of the Northern Filament indicates structures at the same distance from the cluster, as the filament tends to curve around the cavity.

near the southern edge of the map. The eastern one ("Eastern Filament", see Sect. 4.1, Fig. 4) is much more prominent (by a factor of $>2$ with respect to column density, see also Fig. 2, left) than the western one. The Pillars of Creation lie on a filament ${ }^{5}$, which joins the aforementioned Western Filament (see Fig. 4).

The northern half of the M 16 region, just north of the central NGC 6611 cluster, displays a complex and clustered network of filaments. The most coherent filament in this region starts close to the NGC 6611 cluster, connects to the ridge and thereafter heads north-west (see Fig. 4). We hereafter call this filament the "Northern Filament" - see Sect. 4.2.

Interestingly the clear $70 \mu \mathrm{m}$ filaments, seen to the southeast (see Fig. A.1, top-left), which highlight PDR or low column density filaments, are generally perpendicular to the main SE-NW direction of the cloud and the major filaments (see Fig. A.1, top-left). These $70 \mu \mathrm{m}$ emission features are not associated with point like emission of YSOs, but rather to heating/UV ionisation of cloud edges, such as limb brightening/PDRs. These filaments are similar to the striations seen in regions such as Taurus (Palmeirim et al., in prep.).

\subsection{The arch and a potential ridge}

In the individual Herschel images of the Eagle Nebula there is clear evidence of a arch-like structure to the north-west of the NGC 6611 cluster (Figs. 1 and A.1). This arch-like structure is actually composed of two arches, an inner warmer $(\sim 17-20 \mathrm{~K})$ arch, and an outer high column density $\left(\sim 2.0-5.8 \times 10^{22} \mathrm{~cm}^{-2}\right)$ arch. These two arches may in fact be tracing different parts of the same shell-like structure or bubble, as discussed below in Sect. 5.1. These structures are more prominent at the shorter PACS wavelengths. Due to its tendency of tracing density crests, DisPerSE only traces the column density arch (see, Fig. 4).

\footnotetext{
5 Note that DisPerSE identifies a single filament for thee pillars, from the column density crest points. This is simply a limitation of the column density map, as at $37^{\prime \prime}$ resolution only the densest (western most) pillar is detected (see Fig. 2, right).
}

Since the arch appears weak at $8 \mu \mathrm{m}$ (with IRAC; see Indebetouw et al. 2007, Fig. 5), and not as clearly as in our Herschel images (see for example Figs. 1 and A.1 and Sect. 3), it is likely composed of mostly cool material. At the base of the arch is a potential ridge of material. Hill et al. (2011) define a "ridge" as a high-column density $\left(\sim 10^{23} \mathrm{~cm}^{-2}\right)$ super-critical filament dominating its environment that is capable of forming high-mass stars. The most prominent part of the Northern Filament has a column density above $1.0 \times 10^{23} \mathrm{~cm}^{-2}$ over $\sim 2 \mathrm{pc}$. It is not as dominant, i.e., containing most of the material in the vicinity, as the Vela $\mathrm{C}$ ridge or DR 21 ridge (Hill et al. 2011; Hennemann et al. 2012, respectively). This ridge and its star formation content is discussed by White et al. (in prep.).

\section{Impact of the NGC 6611 cluster on the M 16 cloud}

The NGC 6611 cluster is responsible for most of the mechanical and radiative energy incident on the molecular cloud complex around the Eagle Nebula (M 16). Here we assess the dust heating effects in view of constraining the geometry of the complex.

\subsection{The cold Eastern Filament}

The Eastern Filament is a cold prominent filament running east of the NGC 6611 cluster in the column density and dust temperature maps (Figs. 2-4). This southern portion of this filament houses many $70 \mu \mathrm{m}$ sources (see Fig. A.1, top-left), that we interpret as embedded protostars.

In order to determine how the presence of the OB cluster in $\mathrm{M} \mathrm{16}$, in particular its heating effect, has impacted the Eastern Filament, column density and dust temperature profiles were taken along the filament. These profiles were derived using the crest points identified by the DisPerSE algorithm (see Sect. 3.2), and are presented in Fig. 5 (left) as a function of projected distance from the cluster. The dust temperature profile of the Eastern Filament shows a decreasing temperature with 

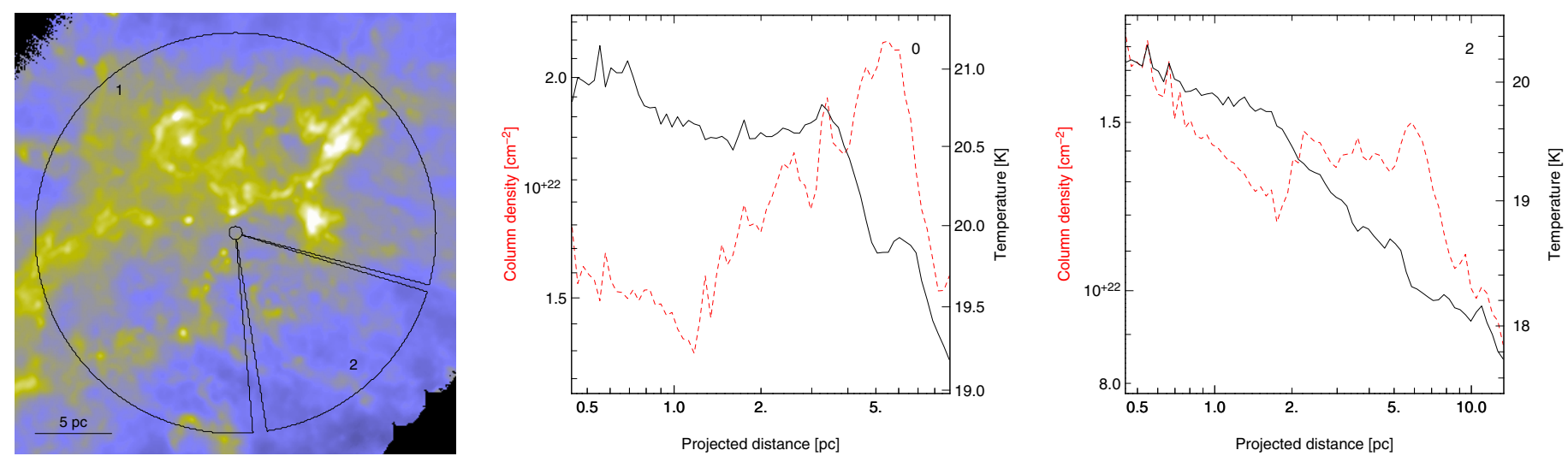

Fig. 6. Left: column density map of NGC 6611 with radial segments as indicated. Segment 1 (middle) contains all of the star formation activity in the region, and segment 2 (right) the cavity carved out by the NGC 6611 cluster. These profiles have been averaged over azimuthal angle. The black solid line is the dust temperature profile, while the red dashed line is the column density profile. More detailed segments for each region inside segment 1 are given in Fig. C.1. Cuts are the median profile in each segment to limit biases introduced by the presence of protostars.

increasing distance from the OB cluster. There are small fluctuations along the temperature profile, which are anti-correlated with column density (see the lower panel of Fig. 5) and thus density enhancements such as star-forming sources located within the filament. Around $\sim 17 \mathrm{pc}$ from the OB cluster the temperature of the Eastern Filament stabilises as it approaches the southern edge of the map. At distances $>17 \mathrm{pc}$, the dust temperature in the Eastern Filament increases with increasing distance as it approaches the Galactic plane. The Eastern Filament has a $\sim 3 \mathrm{~K}$ temperature gradient over its projected length of $\sim 20 \mathrm{pc}$.

To remove the contributions of cold dense sources within the Eastern Filament to the dust temperature we have a applied a de-screening to the data following the relation ${ }^{6}$ by Juvela et al. (Fig. 1; 2011). This relation (green line on Fig. 5) simulates the temperature structure for a filament with a homogeneous density crest, and and while it removes the effects of density enhancements above $N_{\mathrm{H}_{2}}$ ref (where $N_{\mathrm{H}_{2}}$ ref is taken here as $3.2 \times 10^{22} \mathrm{~cm}^{-2}$ ) including, cold dense fragments embedded within the filament, it does not remove the centrally heated protostars (indicated as YSOs in Fig. 5). This column density filtering highlights the effect of the OB cluster on the dust temperature of the Eastern Filament as a whole, the overall trend of which is a decreasing dust temperature with increasing distance from the OB cluster up to a projected distance of $\sim 17 \mathrm{pc}$.

In contrast to the dust temperature, the mean column density profile (Fig. 5, left) appears to remain rather constant with distance from the OB cluster, though it is subject to the aforementioned density enhancements. The strong (and wide) density fluctuation seen at $\sim 20 \mathrm{pc}$ reaching $7 \times 10^{22} \mathrm{~cm}^{-2}$, is located at approximately where the Eastern and Western Filaments appear to meet (see Sect. 3.2).

\subsection{The Northern Filament}

The Northern Filament originates at the eastern part of the NGC 6611 cavity, runs around the cavity and across the potential

\footnotetext{
${ }_{6} T_{\text {corrected }}=T_{\text {measured }}+4.4 \log \left(N_{\mathrm{H}_{2}} / N_{\mathrm{H}_{2} \text { ref }}\right)$; where $N_{\mathrm{H}_{2} \text { ref }}$ is the median column density of the filament. This relation, found in radiative transfer simulations of dense cores, is taken here as an approximation to that which should exist in Plummer-like (cf. Arzoumanian et al. 2011) filaments. A paper is currently being prepared on this topic which examines such a relation in Herschel filaments (Didelon et al., in prep.).
}

ridge (see Sect. 3.3 and White et al., in prep.) before continuing into the northern part of the map (see Fig. 4).

The column density and dust temperature profiles of the Northern Filament are given in Fig. 5 (right). The dust temperature of this filament remains rather constant $(\sim 20 \mathrm{~K})$ around the NGC 6611 cluster, except for a temperature spike at $\sim 3 \mathrm{pc}$ corresponding to a position close to a well known HII region (IRAS 18156-1343). At $\sim 6 \mathrm{pc}$ the dust temperature drops rapidly with increasing distance from the NGC 6611 cluster $(5 \mathrm{~K}$ over $4 \mathrm{pc}$ ), before stabilising at a near constant temperature of $\sim 16 \mathrm{~K}$. This temperature drop is greater than that seen in the Eastern Filament (see Fig. 5, left). This difference is (partly) because the column density profile is less homogeneous than the Eastern Filament, with a large dip in the column density curve due to the presence of the ridge. While the dust temperature used here is a line of sight average for the filament, and the absolute values of the dust temperature are likely to be slightly overestimated as a result, the gradient identified in these Eastern and Northern filaments is still present and robust.

Similarly to the Eastern Filament, a de-screening was applied to the Northern Filament, with $N_{\mathrm{H}_{2}}$ ref, the median column density, taken as $4.4 \times 10^{22} \mathrm{~cm}^{-2}$. After applying this descreening, the dust temperature of the Northern Filament (green line Fig. 5, right) decreases steadily over a much greater distance $(5 \mathrm{~K}$ over $8 \mathrm{pc}$ ). Even after de-screening, the drop in dust temperature is greater than that in the Eastern Filament, which may result from the less homogeneous column density in the Northern Filament or it might also suggest a larger relative inclination along the line of sight, though spectral line (velocity) information is needed to support this idea. Note that at distances $>12 \mathrm{pc}$ the de-screening temperature is much lower $(\sim 2 \mathrm{~K})$ than that without de-screening. This is likely due to the fact that there are very few dense cores in this portion of the filament (see Fig. 2), and thus the de-screening does not completely apply here.

\subsection{NGC 6611 and its immediate surroundings}

In order to determine the extent of the heating from the OB cluster on low column density material $\left(1.5 \times 10^{22} \mathrm{~cm}^{-2}\right.$ or lower, see Fig. 6), PA-averaged radial profiles of both the dust temperature and the column density were taken covering all of the dense star-forming material in the region, as well as the cavity carved out by the NGC 6611 cluster where there is more diffuse 
Table 1. Extent of the heating penetrating of NGC 6611 on the two main filaments identified in the M 16 complex, determined from the dust temperature profiles (Fig. 5).

\begin{tabular}{lccccc}
\hline \hline Name & Colour & $\begin{array}{c}\text { Column } \\
\text { density } \\
\left(\times 10^{22} \mathrm{~cm}^{-2}\right) \\
\text { median }\end{array}$ & $\begin{array}{c}\text { Temper- } \\
\text { ature } \\
(\mathrm{K}) \\
\text { median }\end{array}$ & $\begin{array}{c}\text { Penetration } \\
\text { distance } \\
(\mathrm{pc}) \\
(\text { heating) }\end{array}$ & $\begin{array}{c}\text { Heating } \\
\text { gradient } \\
(\mathrm{K} / \mathrm{pc})\end{array}$ \\
(filaments)
\end{tabular}

Notes. The parameters of the column density and dust temperature derived from probability distribution functions (PDFs) for each sub-region in M 16 (Sect. B and Fig. B.1) as well as their heating distance and gradient are also given (Fig. C.1). The numbers given in parentheses in the last column is the value obtained when a de-screening has been applied (see Sect. 4.1). The asterisk here $\left(^{*}\right)$ indicates that the heating gradient was taken over the steepest portion (3-5 pc), see Fig. C.1.

material. These two radial profiles are presented in Fig. 6, with narrower radial profiles covering the interesting features in this region, e.g., the pillars, given in Fig. C.1. The profiles, which take the median profile in the region rather than the average to limit the inclusion and bias of protostars, were centred at the position of the NGC 6611 cluster.

It is immediately clear (see Fig. 6) that the dust temperature is rather homogeneous close to the NGC 6611 cluster $(\sim 1.5-2 \mathrm{pc})$ but then it drops with increasing distance from the cluster. The dust temperature profile covering most of the emission in the region (Segment 1, Fig. 6) drops $2 \mathrm{~K}$ within $\sim 4 \mathrm{pc}$, and continues to decrease with increasing distance. The dust temperature profile of the cavity (Segment 2) indicates a homogeneous dust temperature close to the cluster $(<2 \mathrm{pc})$, and then a steadily decreasing temperature with increasing distance from the cluster. The column density is anti-correlated with the dust temperature, a result which also applies to the smaller segments presented in Fig. C.1. Each of the segments presented in Fig. C.1 display different dust temperature profiles, suggesting different column densities of material and/or three-dimensional effects.

The extent of heating by the NGC 6611 cluster on the surrounding environment, i.e., the penetration distance, is found to be $3 \mathrm{pc}$ to $15 \mathrm{pc}$ as determined from the dust temperature profiles (see the dust temperature gradient in Figs. 5, 6, C.1), depending on the region (see Table 1). It is not qualitatively different from the penetration measured for $3-6 \times 10^{22} \mathrm{~cm}^{-2}$ filaments (see Sects. 4.1 and 4.2).

\section{Discussion and interpretation}

The Eagle Nebula (M 16) is arguably one of the finest examples of high-mass star formation in the Galaxy, chiefly because of the large number of high-mass stars forming in this region (cf. Hillenbrand et al. 1993) and the remarkable visual effects that this activity has had on the complex (e.g. the Pillars of Creation, Spire, and the "Eagle" in the optical). The Nebula's centre has largely been excavated by the radiation and powerful stellar winds of the OB stars within the central NGC 6611 cluster. This radiation has shaped, and continues to shape, the molecular cloud material, possibly even triggering the formation of new stars and the dissipation of material.

\subsection{The (three-dimensional) structure of the complex}

The three-colour composite image of this region (Fig. 1) shows a very distinct temperature gradient running away from the centre of the cavity carved out by the NGC 6611 cluster. Those regions closest to the cluster are subject to heating by the OB stars, which is reflected in the higher dust temperatures $(+3-5 \mathrm{~K})$ compared with those regions in the more remote $(14-17 \mathrm{pc})$ parts of the cloud, where the heating no longer penetrates. The temperature gradient from the NGC 6611 cluster is similar to that seen in Rosette (Schneider et al. 2010) but over a smaller distance, with a clear drop in temperature within $10 \mathrm{pc}$ of the cluster in all directions (see Figs. 5 and 6).

The M 16 complex displays evidence of a large number of bright rims, which typically occur close to PDRs, especially in the vicinity of the NGC 6611 cluster (see Figs. 2, right and 3 and Sect. 3.2). Whilst a greater investigation into these potential sites of limb-brightening is warranted, we can use the information in these maps to infer the structure relative to the OB cluster (e.g., Minier et al. 2012). Those objects that exhibit signs of bright blue limb-brightening emission at their surface are likely directly receiving incident radiation from the NGC 6611 cluster and are thus at the same distance as the cluster or behind it. There are clear pockets of hot dust (traced by $70 \mu \mathrm{m}$ emission, shown in blue, in Fig. 1) on the southern side of the NGC 6611 cluster, around the pillars, and to the north of the cluster, as well as inside the arch structure (also seen in Fig. 3). This inner $10 \mathrm{pc}$ shell is suggestive of a three-dimensional geometry where the bright $70 \mu \mathrm{m}$ objects, such as the Pillars of Creation, are illuminated by the NGC 6611 cluster and not extinguished by any cloud in its foreground. The location of the Spire and the ridge is less clear. On the other hand, objects such as the MYSO, which do not show evidence of bright rims, and thus incident radiation from the cluster, are clearly in front of the cluster, and closer to us along the line of sight.

An interesting question is raised with respect to the origin of the arch structure (Sect. 3.3 and Fig. 4). Is it actually one structure e.g., created by the expansion of radiation similar to that of a bubble, or is it a consequence of morphology? As noted in Sect. 3.3, two arches are in fact detected at this location and DisPerSE only detects the denser of these. Figures 1 and 2 (right) suggest that the inner warmer arch is closer to the cluster, directly influenced by its heating, whilst the outer higher column density arch is likely above (i.e., closer to us along the line of sight) and away from the cluster. This suggests some sort of curved morphology, where the two arches are in fact two sides of a three dimensional shell-like or bubble-like structure, with the near (denser arch) and far (warmer arch) curved sides of the shell/bubble appearing as two arches in two dimensions.

Two prominent filaments are detected in the M 16 complex, a cool dense Eastern Filament (see Sect. 4.1) which spans almost $25 \mathrm{pc}$, and the Northern Filament (see Sect. 4.2) which extends across the ridge, at the western side of the arch structure, and up to $\sim 15$ pc. Figures 2 (left) and 3 indicate a clear branch towards the southern end of the Eastern Filament, at $\sim 15 \mathrm{pc}$ from the origin (see Fig. 5, left), as discussed in Sect. 4.1. DisPerSE (Sect. 3.2) actually detects the much weaker (with respect to column density - Fig. 2) western component of this branch (Fig. 4), which extends up to the base of the Pillars of Creation. If we were to extrapolate or extend this western filament, it would 
continue to join the Northern Filament at the approximate location of, and along the same line of sight as, the MYSO. In this scenario, the Eastern Filament has remained largely undisturbed whilst the Western Filament has undergone a dramatic transformation. Once the home of the NGC 6611 cluster, it would have been destroyed as the cluster accumulated mass and formed the high-mass OB stars seen in existence today. The strong ionising winds from the OB stars themselves would have carved out a cavity in this filament, and eradicated any existence of the continuation of this Western Filament. Small grains associated with this Western Filament are still present and emit strongly at $70 \mu \mathrm{m}$ (Fig. A.1, top-left).

\subsection{The heating effect of the NGC 6611 cluster}

The high-mass star cluster NGC 6611 has noticeably impacted, in terms of heating, the M 16 complex as a whole. The dust temperature profiles (Figs. 5 and 6) clearly show the effect and the extent of this impact, i.e., how much and how far it has affected the temperature in all directions. Despite the fact that the dust temperature measured at the crest (Fig. 5) or averaged within segments (Fig. 6) obviously depends on the column density of the material traced, the penetration distances do not seem very different from the diffuse emission of segment 1 $\left(<N_{\mathrm{H}_{2}}>10^{22} \mathrm{~cm}^{-2}\right)$ to the high column density emission of the Northern Filament $\left(<N_{\mathrm{H}_{2}} \gtrsim 6 \times 10^{22} \mathrm{~cm}^{-2}\right)$. Our analysis (see Figs. 5 and 6 and Table 1 ) has shown that NGC 6611 is able to heat the M 16 cloud to a projected distance of $\sim 8-10 \mathrm{pc}$. The M 16 cloud displays $\sim 1.5-5 \mathrm{~K}$ temperature variations, corresponding to heating gradients of $0.25-0.6 \mathrm{~K} / \mathrm{pc}$, with an average value of $\sim 0.3 \mathrm{~K} / \mathrm{pc}$. The dust temperature profiles of the Eastern and Northern Filaments (Fig. 5) indicate that NGC 6611 is able to heat, by $3-5 \mathrm{~K}$, star-forming sites $\left(>10^{5} \mathrm{~cm}^{-3}\right.$ dense cores) approximately up to $10-11 \mathrm{pc}$ into each filament. While the dust temperature and column density gradients are robust, the absolute values of these quantities are subject to line of sight effects.

It is clear from the radial profiles (Fig. 6) that the heating effect of NGC 6611 is not the same in all directions. These differences would be expected if the NGC 6611 cluster is in fact at the same distance as only some of these regions (as suggested in Sect. 5.1). Alternatively, the ionisation and heating depths of the cluster is simply dependent on the amount of material in the way, such that high column density regions will block the radiation better than low column density regions in which the radiation more easily penetrates.

Herschel observations have shown that interstellar filaments act as the birthplace of star formation. In M 16, we have shown that interstellar filaments can largely be influenced by heating, such as that coming from the nearby high-mass NGC 6611 cluster (e.g., Fig. 5). Such external heating is often not considered when deriving the evolutionary status of a core from its farinfrared SED. Evolution/age proxies such as $L_{\text {submm }} / L_{\text {bol }}$ (André et al. 2000) or $T_{\text {bol }}$ (Myers \& Ladd 1993) for evolutionary diagrams have been recently extrapolated to massive dense cores (e.g. Motte \& André 2001). These parameters should be taken with caution, since massive dense cores generally form in molecular cloud complexes associated with OB clusters. By ignoring this effect, $T_{\text {bol }}$ will appear cooler at larger distances from the cluster, and hotter closer to the cluster. Similarly, the $L_{\text {submm }} / L_{\text {bol }}$ ratio of a protostellar core will be larger further from the cluster than closer to it. Inferring evolutionary trends from these parameters, one would then naturally find more evolved protostellar dense cores closer to the cluster than further away from it, giving the (incorrect) impression of triggered star formation. In order to avoid these biases, the effects of external heating on interstellar filaments should be considered when determining the evolutionary state of a star-forming core, especially in high-mass star-forming regions.

As an example, a $\sim 6 \mathrm{~K}$ dust temperature gradient has been found for massive dense cores forming in the Rosette molecular cloud under the influence of the NGC 2244 cluster (Motte et al. 2010). Even after subtracting a possible $3 \mathrm{~K}$ gradient coming from the different temperatures of the cloud filaments inside which prestellar cores have formed and evolved into protostars, the remaining $3 \mathrm{~K}$ could still argue for an age trend for these YSOs, as originally suggested by Schneider et al. (2010).

Our Herschel data of M 16 do not provide any evidence for a supernova remnant, as suggested by Flagey et al. (2011). Our maps (Fig. 2, right) indicate dust temperatures in M 16 that are consistent with other high-mass star-forming regions (Nguyen Luong et al. 2011; Hill et al. 2011). Flagey et al. (2011), with their shorter wavelength Spitzer data, were more sensitive to hot dust which is being shattered by a supernova remnant. Based on this comparison alone, we are unable to support or refute this claim based on our Herschel data, which are more sensitive to colder dust. However, young supernova remnants such as SN 1987A (Matsuura et al. 2011) and Cassiopeia A (Barlow et al. 2010) have been readily observed by Herschel, as have much older supernova remnants e.g. W44. SN 1987A is seen as a point source with PACS and as extended emission at increasingly longer Herschel wavelengths, while Cas A is well traced at all Herschel wavelengths, as well as at $24 \mu \mathrm{m}$ with Spitzer. The fact that we don't see evidence of such a supernova remnant in our individual Herschel images, and that we see no evidence of impact on the column density distribution around the NGC 6611 cluster, as well as the absence of emission seen with Spitzer (e.g. Flagey et al. 2011; Indebetouw et al. 2007) does not favour the presence of a supernova remnant in this region.

Acknowledgements. T.H. is supported by a CEA/Marie-Curie Eurotalents Fellowship. D.E., D.P., K.L.J.R., and E.S. are funded by an ASI fellowship under contract numbers $\mathrm{I} / 005 / 11 / 0$ and I/038/08/0. The authors wish to thank Daniel Price for comments and suggestions on an earlier version of the manuscript. We'd also like to thank Christophe Carreau (ESA) for working his magic with Fig. 1. Part of this work was supported by the ANR (Agence Nationale pour la Recherche) project "PROBeS", number ANR-08-BLAN-0241. This work has made use of the Yorick freeware package (see http://yorick. sourceforge. net/). Figure A.1 was generated using APLpy, an open-source plotting package for Python hosted at http://aplpy.github.com. SPIRE has been developed by a consortium of institutes led by Cardiff Univ. (UK) and including: Univ. Lethbridge (Canada); NAOC (China); CEA, LAM (France); IFSI, Univ. Padua (Italy); IAC (Spain); Stockholm Observatory (Sweden); Imperial College London, RAL, UCL-MSSL, UKATC, Univ. Sussex (UK); and Caltech, JPL, NHSC, Univ. Colorado (USA). This development has been supported by national funding agencies: CSA (Canada); NAOC (China); CEA, CNES, CNRS (France); ASI (Italy); MCINN (Spain); SNSB (Sweden); STFC, UKSA (UK); and NASA (USA). PACS has been developed by a consortium of institutes led by MPE (Germany) and including UVIE (Austria); KU Leuven, CSL, IMEC (Belgium); CEA, LAM (France); MPIA (Germany); INAF-IFSI/OAA/OAP/OAT, LENS, SISSA (Italy); IAC (Spain). This development has been supported by the funding agencies BMVIT (Austria), ESA-PRODEX (Belgium), CEA/CNES (France), DLR (Germany), ASI/INAF (Italy), and CICYT/MCYT (Spain).

\section{References}

Allen, L. E., Burton, M. G., Ryder, S. D., Ashley, M. C. B., \& Storey, J. W. V. 1999, MNRAS, 304, 98

Anderson, L. D., Zavagno, A., Deharveng, L., et al. 2012, A\&A, 542, A10

André, P., Ward-Thompson, D., \& Barsony, M. 2000, Protostars and Planets IV, 59

André, P., Men'shchikov, A., Bontemps, S., et al. 2010, A\&A, 518, L102

André, P., Men'shchikov, A., Könyves, V., \& Arzoumanian, D. 2011, in IAU

Symp., 270, ed. J. Alves, B. G. Elmegreen, J. M. Girart, \& V. Trimble, 255

Arzoumanian, D., André, P., Didelon, P., et al. 2011, A\&A, 529, L6 
Ballesteros-Paredes, J., Hartmann, L. W., Vázquez-Semadeni, E., Heitsch, F., \& Zamora-Avilés, M. A. 2011, MNRAS, 411, 65

Barlow, M. J., Krause, O., Swinyard, B. M., et al. 2010, A\&A, 518, L138

Belikov, A. N., Kharchenko, N. V., Piskunov, A. E., \& Schilbach, E. 2000, A\&A, 358,886

Bernard, J.-P., Paradis, D., Marshall, D. J., et al. 2010, A\&A, 518, L88

Bohlin, R. C., Savage, B. D., \& Drake, J. F. 1978, ApJ, 224, 132

Bontemps, S., André, P., Könyves, V., et al. 2010, A\&A, 518, L85

Cesarsky, C. J., Abergel, A., Agnese, P., et al. 1996, A\&A, 315, L32

Dufton, P. L., Smartt, S. J., Lee, J. K., et al. 2006, A\&A, 457, 265

Federrath, C., Roman-Duval, J., Klessen, R. S., Schmidt, W., \& Mac Low, M.-M. 2010, A\&A, 512, A81

Flagey, N., Boulanger, F., Noriega-Crespo, A., et al. 2011, A\&A, 531, A51

Garay, G., \& Lizano, S. 1999, PASP, 111, 1049

Giannini, T., Elia, D., Lorenzetti, D., et al. 2012, A\&A, 539, A156

Goldsmith, P. F., Bergin, E. A., \& Lis, D. C. 1997, ApJ, 491, 615

Griffin, M. J., Abergel, A., Abreu, A., et al. 2010, A\&A, 518, L3

Gvaramadze, V. V., \& Bomans, D. J. 2008, A\&A, 490, 1071

Hennemann, M., Motte, F., Bontemps, S., et al. 2010, A\&A, 518, L84

Hennemann, M., Motte, F., Schneider, N., et al. 2012, A\&A, in press, DOI: 10.1051/0004-6361/201219429

Hester, J. J., Scowen, P. A., Sankrit, R., et al. 1996, AJ, 111, 2349

Hill, T., Pinte, C., Minier, V., Burton, M. G., \& Cunningham, M. R. 2009, MNRAS, 392, 768

Hill, T., Longmore, S. N., Pinte, C., et al. 2010, MNRAS, 402, 2682

Hill, T., Motte, F., Didelon, P., et al. 2011, A\&A, 533, A94

Hillenbrand, L. A., Massey, P., Strom, S. E., \& Merrill, K. M. 1993, AJ, 106, 1906

Indebetouw, R., Robitaille, T. P., Whitney, B. A., et al. 2007, ApJ, 666, 321
Juvela, M., \& Ysard, N. 2011, ApJ, 739, 63

Juvela, M., Ristorcelli, I., Pelkonen, V.-M., et al. 2011, A\&A, 527, A111

Kainulainen, J., Beuther, H., Henning, T., \& Plume, R. 2009, A\&A, 508, L35

Linsky, J. L., Gagné, M., Mytyk, A., McCaughrean, M., \& Andersen, M. 2007, ApJ, 654, 347

Matsuura, M., Dwek, E., Meixner, M., et al. 2011, Science, 333, 1258

Minier, V., Tremblin, P., Hill, T., et al. 2012, A\&A, submitted

Molinari, S., Swinyard, B., Bally, J., et al. 2010, A\&A, 518, L100

Motte, F., \& André, P. 2001, A\&A, 365, 440

Motte, F., Zavagno, A., Bontemps, S., et al. 2010, A\&A, 518, L77

Myers, P. C., \& Ladd, E. F. 1993, ApJ, 413, L47

Nguyen Luong, Q., Motte, F., Hennemann, M., et al. 2011, A\&A, 535, A76

Ott, S. 2010, in Astronomical Data Analysis Software and Systems XIX, ed.

Y. Mizumoto, K.-I. Morita, \& M. Ohishi, ASP Conf. Ser., 434, 139

Peretto, N., Andre, P., Konyves, V., et al. 2012, A\&A, 541, A63

Pilbratt, G. L., Altieri, B., Blommaert, J. A. D. L., et al. 1998, A\&A, 333, L9

Pilbratt, G. L., Riedinger, J. R., Passvogel, T., et al. 2010, A\&A, 518, L1

Poglitsch, A., Waelkens, C., Geis, N., et al. 2010, A\&A, 518, L2

Pound, M. W. 1998, ApJ, 493, L113

Roussel, H. 2012, A\&A, submitted

Schneider, N., Motte, F., Bontemps, S., et al. 2010, A\&A, 518, L83

Schneider, N., Csengeri, T., Hennemann, M., et al. 2012, A\&A, 540, L11

Sousbie, T. 2011, MNRAS, 414, 350

Sousbie, T., Pichon, C., \& Kawahara, H. 2011, MNRAS, 414, 384

Urquhart, J. S., White, G. J., Pilbratt, G. L., \& Fridlund, C. V. M. 2003, A\&A, 409, 193

White, G. J., Nelson, R. P., Holland, W. S., et al. 1999, A\&A, 342, 233

Wolff, S. C., Strom, S. E., Dror, D., \& Venn, K. 2007, AJ, 133, 1092

Zavagno, A., Russeil, D., Motte, F., et al. 2010, A\&A, 518, L81 


\section{Appendix A: Herschel images of M 16}

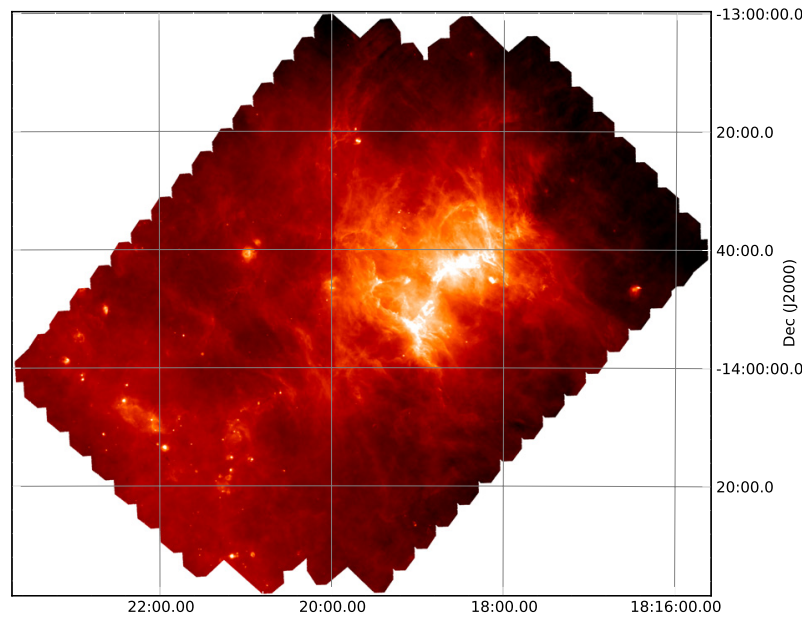

RA (2000)

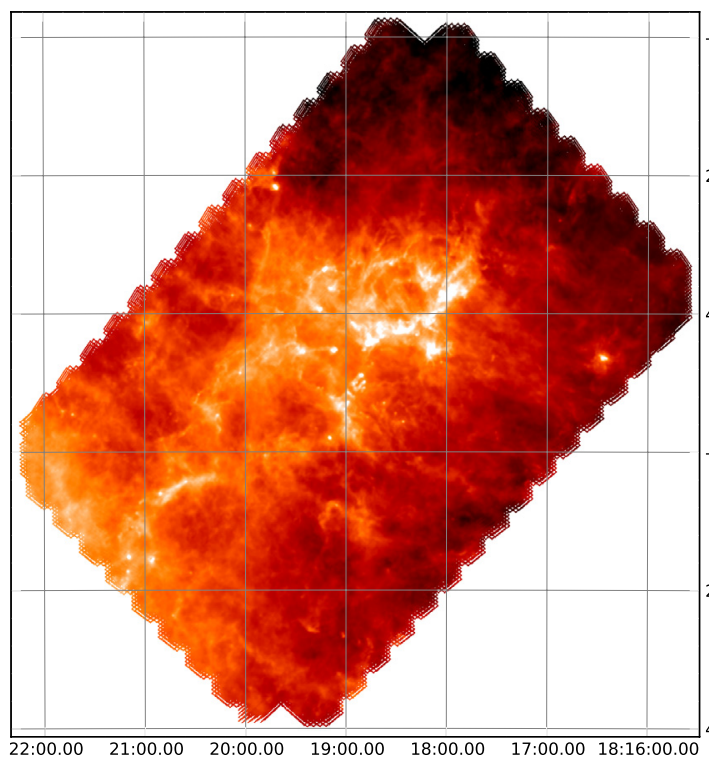

RA (2000)

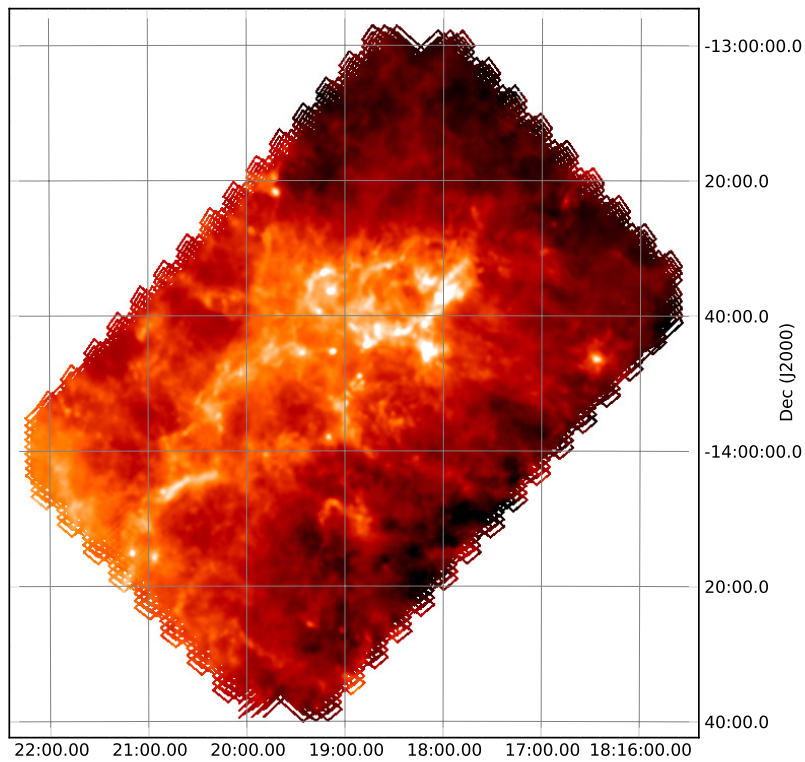

RA (J2000)

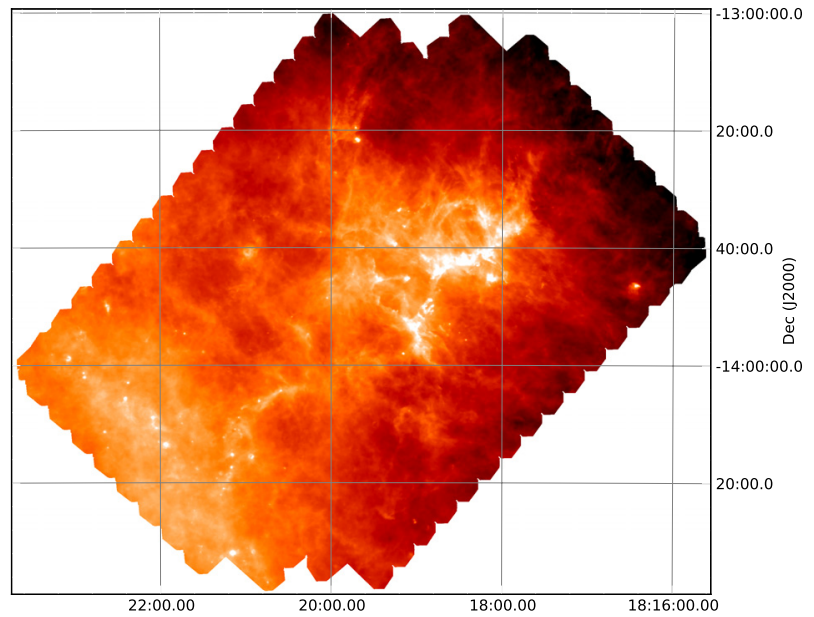

RA (j2000)

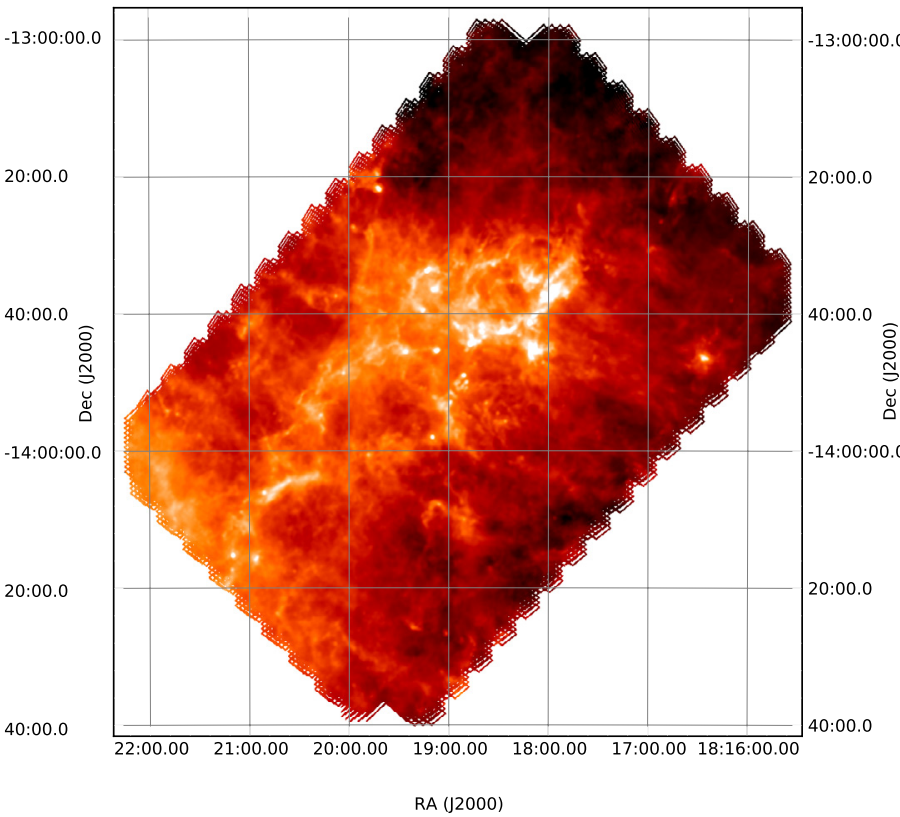

Fig. A.1. The 5 Herschel wavebands of M 16, from top to bottom, 70, 160, 250, 350 and $500 \mu \mathrm{m}$. Note also the extended PACS coverage compared with SPIRE (see Sect. 2). The observed level of cirrus noise in M 16 is $\sim 20 \mathrm{mJy}$ and $200 \mathrm{mJy}$ at $\lambda=70$ and $160 \mu \mathrm{m}$, respectively and 2,1 and $1 \mathrm{Jy}$ at the SPIRE 250, 350 and $500 \mu \mathrm{m}$ bands. At shorter wavelengths only those warmer objects, such as protostars and HII regions are seen. At longer wavelengths Herschel detects cold, deeply embedded filaments and the progenitors of high-mass stars. 
T. Hill et al.: Herschel's perspective of heating on the Eagle Nebula
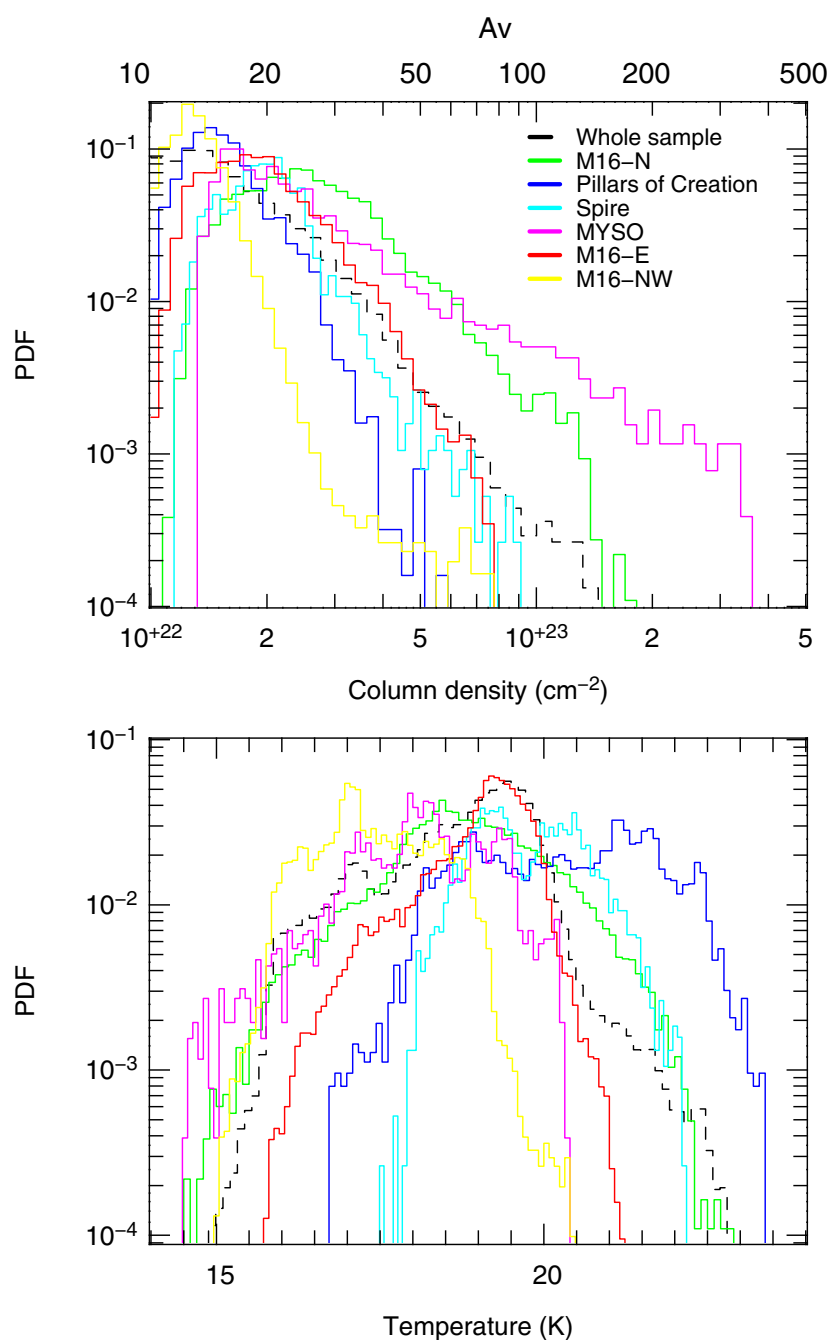

Fig. B.1. PDFs of column density (top) and temperature (bottom) with the sub-regions as defined in Fig. 2. Only the high column density material that we are interested in $>10$ mag is shown.

\section{Appendix B: Probability distribution functions}

To examine the dust temperature and column density structure in the entire M 16 region probability distribution functions (PDFs) were derived (Fig. B.1). Column density PDFs have been used by many authors to characterise cloud structure based on star formation activity (Kainulainen et al. 2009; Federrath et al. 2010). The column density in M 16 spans almost two orders of magnitude $\left(5.0 \times 10^{21}-3.7 \times 10^{23} \mathrm{~cm}^{-2}\right)$, whilst the dust temperature ranges from $14.5 \mathrm{~K}$ to $23.5 \mathrm{~K}$. The highest temperature in the maps is recorded directly west of the base of the Pillars of Creation and thus south of the NGC 6611 cluster.

In order to investigate the variation of PDF profiles within M 16, we segregated the complex into six sub-regions (see Fig. 2, left), according to the prominent structures or cloud features impacted by the NGC 6611 cluster. These sub-regions include: the main component of material, corresponding to the Eagle's wings in optical images and the arch structure (hereafter referred to as the "M 16-N" sub-region; green in Fig. B.1), the Pillars of Creation (blue), Spire (cyan), the MYSO detected by Indebetouw et al. (magenta 2007), the eastern portion of the map (hereafter "M 16-E"; red), and the northern periphery (hereafter "M 16-NW"; yellow). The MYSO is treated as a separate sub-region due to its high column density tail, to prevent this MYSO from skewing the PDFs of other sub-regions. While the column density PDF of this MYSO should be treated with caution, its inclusion here allows comparison with the other sub-regions with respect to dust temperature.

The column density PDFs are reliable for the larger regions (e.g., M 16-N, M 16-E, M 16-NW) above $A_{\mathrm{V}}>10 \mathrm{mag}^{7}$, whilst the smaller regions (e.g., Pillars of Creation, Spire and the MYSO) are reliable only above $A_{\mathrm{V}}>20 \mathrm{mag}$. We are only concerned with higher column density ( $>20 \mathrm{mag}$ ) here. The M 16-N, Spire and M 16-E sub-regions have similar column density slopes (around $A_{\mathrm{V}}>30 \mathrm{mag}$ ). The M 16-N and MYSO sub-region have high column density tails, similar to that seen in the Vela C Centre-Ridge sub-region (Hill et al. 2011), and consistent with regulation by self-gravity (Ballesteros-Paredes et al. 2011).

The Pillars of Creation sub-region has a column density PDF that resembles a log-normal shape which has been seen in clouds which are initially quiescent and where turbulence is likely the main shaping mechanism (Kainulainen et al. 2009). Note, however that feedback effects, such as those from a nearby OB cluster, have yet to be factored into turbulence simulations from which model PDFs are drawn. The suggestion of turbulence here is consistent with the observations of Pound $\left(1998,{ }^{12} \mathrm{CO}\right.$, ${ }^{13} \mathrm{CO}$ and $\mathrm{C}^{18} \mathrm{O}$ ) who found that the Pillars of Creation are not gravitationally bound and are being ripped apart.

The sub-region hosting the Pillars of Creation (Fig. B.1, blue) attains higher dust temperatures than the other sub-regions, including that of the Spitzer identified MYSO. This difference shows that the NGC 6611 cluster has had more of an impact on the cloud temperature than the current star formation process. The M 16-N and Spire sub-regions have similar dust temperature PDF slopes $(>21 \mathrm{~K})$, which likely reflects their similar distance from the NGC 6611 cluster, though the M 16-N sub-region contains colder gas in the northern part of the region, including the ridge itself. The M 16-NW and M 16-E sub-regions span only small ranges in temperature. The median column density and dust temperature for each sub-region, determined from the PDFs, are given in Table 1.

It is interesting to note that while the M 16-E and Spire subregions have similar column density PDF profiles, they display quite different dust temperature PDF profiles. This suggests that they are both a part of the same cloud structure, but that the Spire is closer to and thus more readily externally heated by the NGC 6611 cluster than M 16-E. The three sub-regions closest to the OB cluster (Pillars of Creation, Spire, MYSO) have similar dust temperature profiles, but quite different column densities.

There is no clear trend in the column density PDFs to suggest or represent an effect of the winds or radiation from the NGC 6611 cluster on the sub-regions. The median temperature of each region correlates closely with the projected distance from the cluster, suggesting no strongly unbalanced projection effects for the cloud surrounding the NGC 6611 cluster.

The probability distribution functions (Fig. B.1) confirm that the column density in each sub-region in M 16 is indeed different, which influences the extent of the heating effect of the NGC 6611 cluster on each sub-region, as seen in the dust temperature PDFs. At zero-order and above $A_{\mathrm{V}}>15 \mathrm{mag}$ there is no difference seen in the structure of the cloud with distance from the NGC 6611 cluster. That is, the column density PDFs of sub-regions near to the cluster, and thus heavily impacted,

Where $N_{\mathrm{H}_{2}}=1 A_{\mathrm{V}} \times 10^{21} \mathrm{~cm}^{-2} \mathrm{mag}^{-1}$ (Bohlin et al. 1978). 
A\&A 542, A114 (2012)
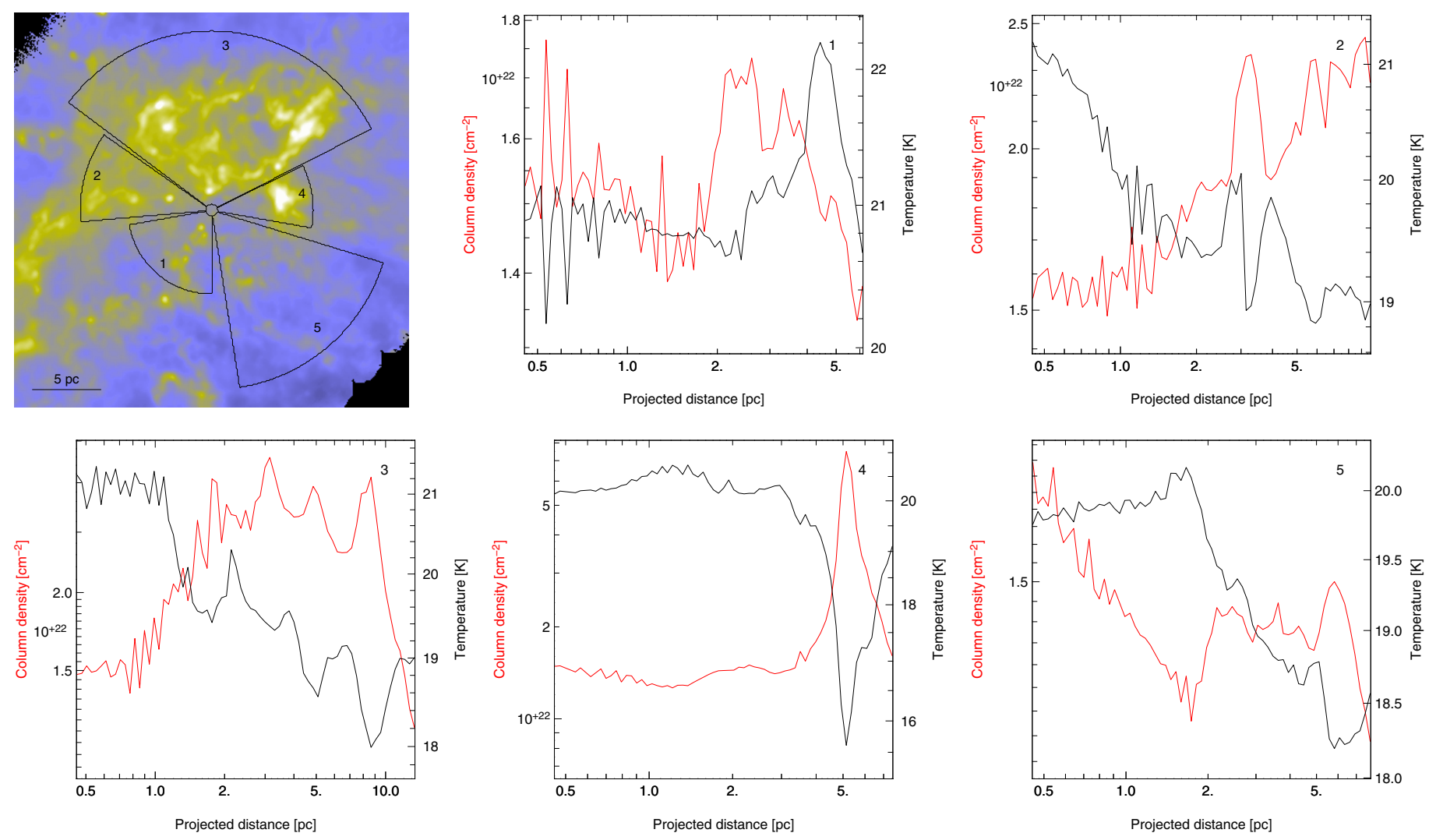

Fig. C.1. Upper left: column density map of NGC 6611 with radial segments as indicated. Upper-middle to lower-right: dust temperature (black) and column density (red) PA-averaged radial profiles in each segment. Segments are arranged clockwise starting with the Pillars of Creation (upper-middle) to the cavity region (lower-right) as identified by numbers. The profiles are the median values with projected distance in each segment to avoid biases by the presence of protostars. The radial lengths of each segment were defined by a drop in column density (as seen in the upper-left panel).

and those in more remote portions of the map, display similar column density PDF slopes.

It is also worth noting, that though normalised, the Pillars of Creation, Spire and MYSO sub-regions are comprised of a small number of pixels compared with the other sub-regions in the complex, which could thus affect their interpretation.

\section{Appendix C: Detailed radial segments around NGC 6611}

The radial profiles of more detailed segments (than Fig. 6) around the NGC 6611 cluster, including the Pillars of Creation, can be found in Fig. C.1. 